
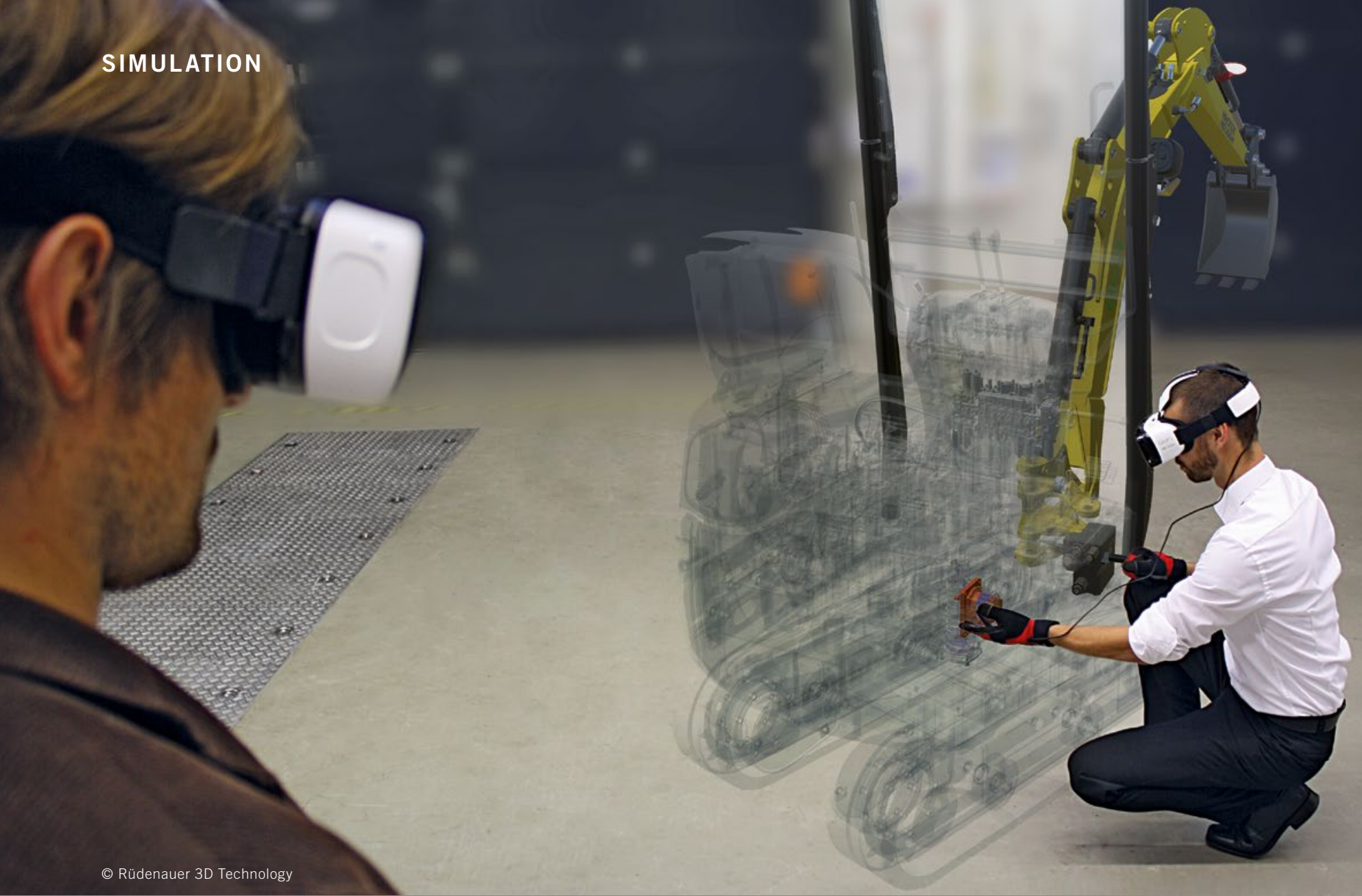

\title{
Vernetzung und 3-D-Technologien im digitalen Unternehmen der Zukunft
}

Wer heutzutage in der Industrie eine Maschine immersiv in 3-D sehen möchte, begibt sich in einen 3-D-Raum. Bekanntester Vertreter darunter ist die sogenannte CAVE. Der enorme Investitionsaufwand in Millionenhöhe sowie Einschränkungen des 3-D-Erlebnisses in Gruppen-Sessions schränken hier die Verbreitung auf große Konzerne und wenige Hochschulen ein. Als Spin-Off vom Lehrstuhl für Mobile Arbeitsmaschinen des Karlsruher Instituts für Technologie (KIT) macht die Rüdenauer 3D Technology GmbH mit ihrer Lösung das immersive 3-D-Produkterlebnis wirtschaftlich verfügbar und unterstützt mit diesem technischen Fortschritt die Digitalisierung der Industrie. Zukünftig können mobile Maschinen virtuell im Cross Connected HoloDeck unter in Echtzeit simulierten Randbedingungen erlebt, getestet und sogar produziert werden - und das kollaborativ und werksübergreifend. 


\section{HERAUSFORDERUNG DER PRODUKTENTWICKLUNG}

Der Erfolg einer Produktentwicklung lässt sich, genau wie für jedes klassische Projekt, anhand der Erfolgsdimensionen des magischen Dreiecks messen, welches durch die zum Unternehmenserfolg benötigten Qualitäts-, Kosten- und Zeitziele beschrieben wird, BILD 1. Dabei gilt es, die steigenden Kundenerwartungen bezüglich der Qualität des Produkts, die anfallenden Kosten der Produktentwicklung, welche durch zunehmenden Wettbewerbsdruck nach oben begrenzt werden, und die sogenannte Time-toMarket, das heißt die Zeit bis zur Markteinführung des Produkts, zu optimieren [2]. Das Fraunhofer-Institut für Systemund Innovationsforschung ISI [3] untergliedert die Treiber, beziehungsweise die Entwicklungen in den Rahmenbedingungen der Produktentwicklung und des Markts in folgende vier Hauptgruppen:

- Globalisierung

- technischer Wandel

- Umweltaspekte

- Mensch.

Unter dem Einfluss dieser Treiber haben sich in der Produktentwicklung Trends entwickelt, welche die Erreichung der teilweise konträren, oben genannten Ziele ermöglichen sollen.

\section{KOLLABORATION ALS ANTWORT AUF DIE HERAUSFORDERUNGEN DER PRODUKTENTWICKLUNG}

Als erfolgreiches Reaktionsmuster, um der Komplexität zu begegnen, haben sich Maßnahmen zur unternehmensübergreifenden Kollaboration sowie der virtuellen Produktentwicklung erwiesen. „Collaboration requires a team of individuals to work on tasks that not only have shared resources (as in coordination) and shared outcomes (as in cooperation), but most importantly, a shared common goal“ [4]. Diese Definition des Begriffs Kollaboration ergänzt den Begriff der Koordination, der den Zugriff auf gemeinsame Ressourcen beschreibt, und den Begriff der Kooperation, der das Teilen eines gemeinsamen Ergebnisses in den Vordergrund stellt, um das Arbeiten des Teams an einem gemeinsamen Ziel.

In der Automobilindustrie ist seit Jahren der Trend zur Kollaboration in der Produktentwicklung zu beobachten. Entwicklungsumfänge des Erstausrüsters
(OEM) werden an die Zulieferer ausgelagert. Einer aktuellen Studie der Unternehmensberatung Oliver Wyman und des Verbands der Automobilindustrie (VDA) zufolge, wird sich diese Entwicklung weiter verstärken. Insgesamt wird laut FAST-Studie der Anteil der OEMs an der globalen Wertschöpfung bezüglich Forschung und Entwicklung von heute 60 auf 47 \% im Jahr 2025 sinken, während sich der Anteil der Zulieferer von 32 auf 36 \% erhöht und die EngineeringDienstleister ihren Anteil von 9 auf $17 \%$ fast verdoppeln [5], BILD 2. Beweggründe für diese Entwicklung können aus den oben genannten Treibern Globalisierung, Technik, Umweltproblematik und Mensch abgeleitet werden.

In der Offhighway-Branche existieren zum Teil hoch individualisierte und vernetzte Innovationszyklen. Diese sind stark vom internationalen Kunden- beziehungsweise Marktsegment abhängig. Eine wesentliche Herausforderung liegt darin, im globalen Engineering die Anforderungen des lokalen Markts in einer Plattformstrategie zu berücksichtigen [6]. Moderne Entwicklungsansätze, wie zum Beispiel die auf Kundenwert orientierte Entwicklungsmethode Design-to-Value [7], unterstreichen den Bedarf nach einer konsequenten und iterativen Einbindung

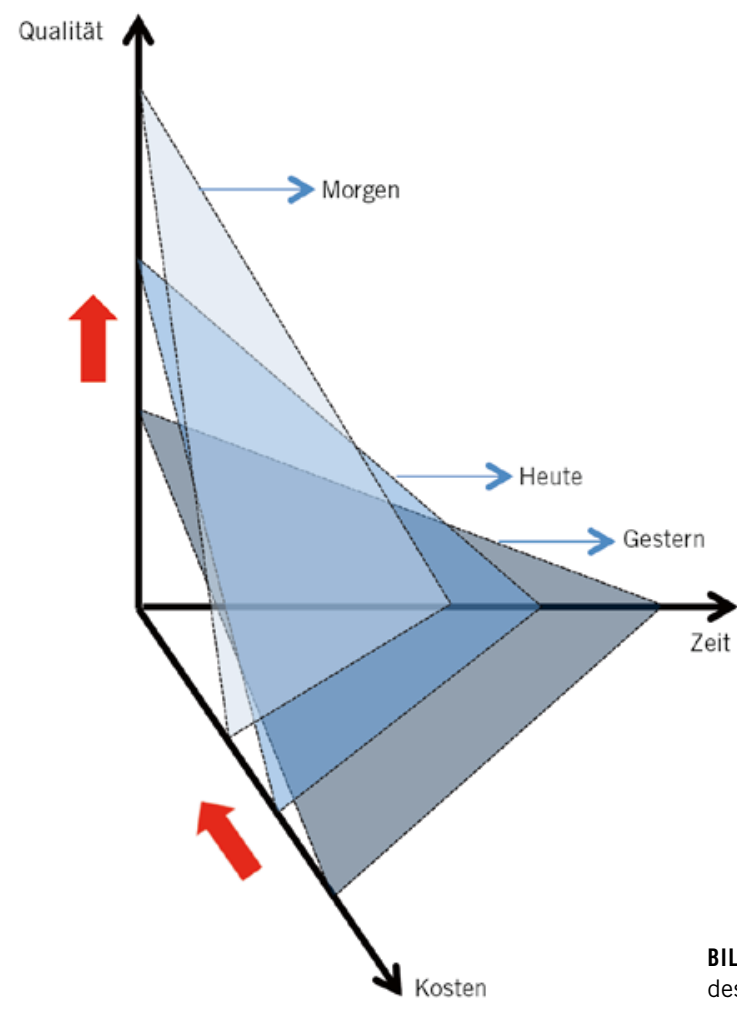

AUTOREN

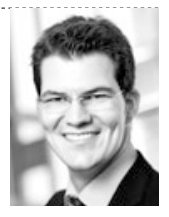

Dipl.-Ing. Andreas Rüdenauer ist Gründer und Geschäftsführer der Rüdenauer 3D Technology $\mathrm{GmbH}$ in Karlsruhe.

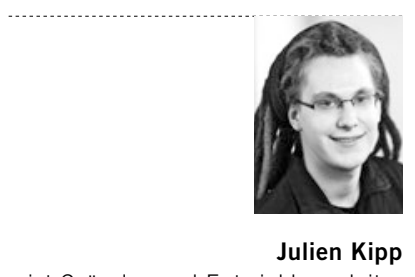

ist Gründer und Entwicklungsleiter der Rüdenauer 3D Technology $\mathrm{GmbH}$ in Karlsruhe.

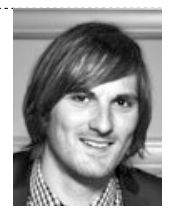

Dr.-Ing. Martin Scherer ist Oberingenieur am Lehrstuhl für Mobile Arbeitsmaschinen des Karlsruher Instituts für Technologie (KIT).

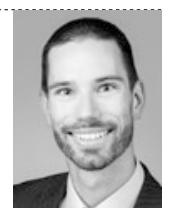

Dipl.-Ing. Bernhard Jahnke war akademischer Mitarbeiter am

Lehrstuhl für Mobile Arbeitsmaschinen des Karlsruher Instituts für Technologie (KIT). 


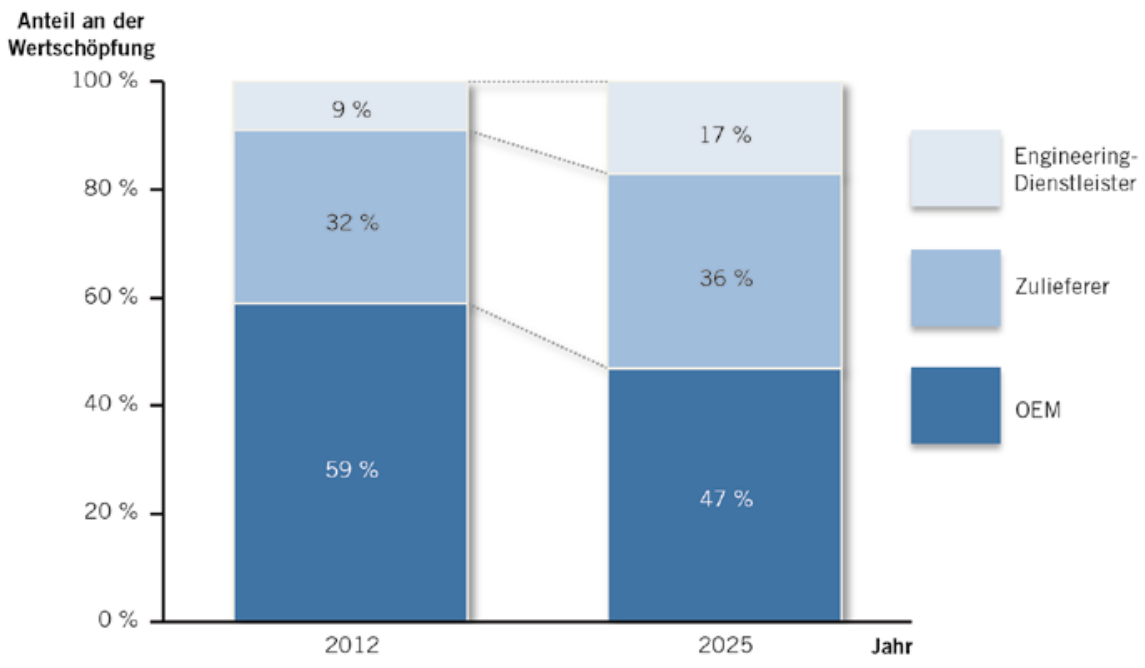

BILD 2 Wertschöpfungsverlagerung in der Automobilindustrie (C KIT)

aller Stakeholder über den Entwicklungsprozess hinweg vom Zulieferer bis hin zum Kunden. Auf diese Weise können am Markt akzeptierte Produkte wettbewerbsfähig aufgestellt werden.

\section{CROSS CONNECTED HOLODECK}

Das Cross Connected HoloDeck bietet hierbei erstmalig die Möglichkeit, standortübergreifende Teams kostengünstig und effizient an ein virtuelles Produkt zu versetzen, um gemeinsam eine Maschine, deren Funktionen sowie ihre Wirkung auf das System simulationsgestützt interaktiv zu erleben, BILD 3. Die Nutzer werden mittels 3-D-Virtual-Reality-Brille und Datenhandschuh direkt an das virtuelle
Fahrzeug versetzt. Dies eröffnet von der Entwicklung bis hin zur Schulung ein hohes Potenzial zur Kosteneinsparung.

Kerninnovation der Software-Plattform Cross Connected ist die Vernetzung vorhandener Daten aus dem 3-D-CADBereich mit Maschinenverhaltensdaten. Verhaltensdaten können hierbei Simulationsdaten aus der Produktentwicklung bis hin zu Mess- und Sensordaten direkt aus der Maschine sein. Ein virtueller, funktional erlebbarer Prototyp einer Maschine wird somit per Mausklick aus den im Unternehmen vorhandenen Daten erzeugt. Ebenso sind Varianten der Maschine auf einfache Weise darstellbar. Darüber hinaus können Produktionsprozesse der Maschine quasi unter Realbedingungen

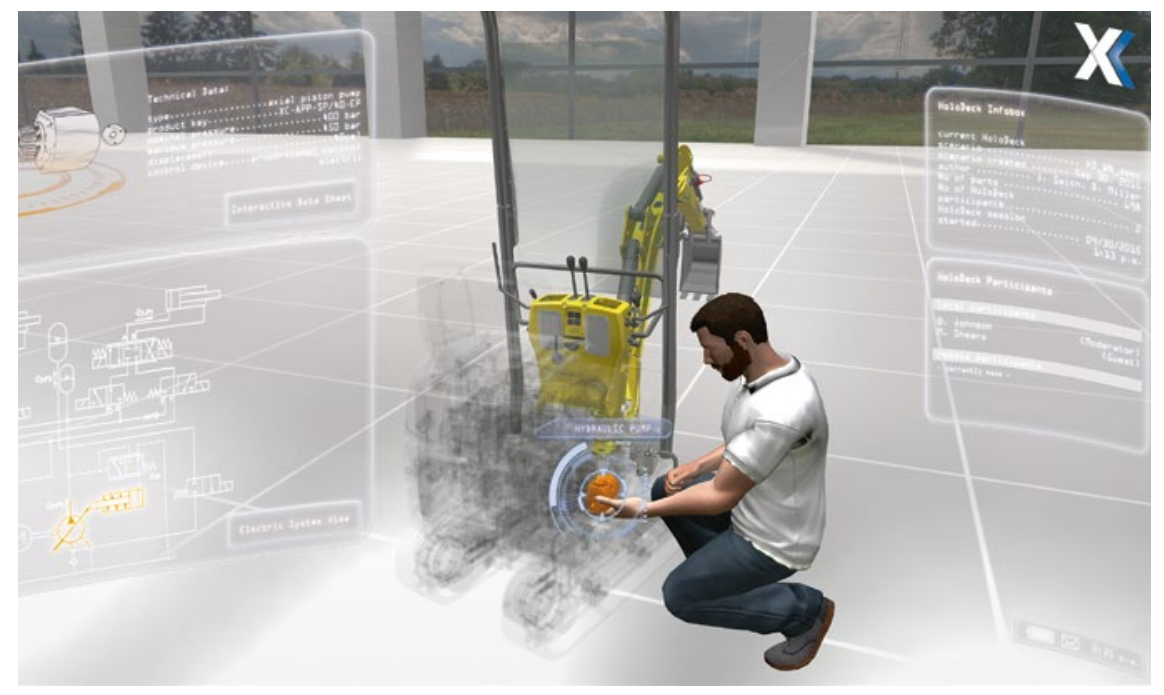

BILD 3 Virtuelle Szene im Cross Connected HoloDeck (@ Rüdenauer 3D Technology) dynamisch im HoloDeck erprobt werden. Eine konsequente Ausrichtung auf Standardschnittstellen ermöglicht automatisierte Workflows zum Import von Konstruktionsdaten sowie von Verhaltensdaten. Innerhalb kürzester Zeit können bestehende Daten in das Holodeck eingebunden und für die Arbeit im Team nutzbar gemacht werden.

Neben der Software-Plattform trägt die Hardware-Ausstattung des Holodecks zum wirtschaftlichen Einsatz in der Industrie bei, BILD 4. Von stationären 3 -D-Kameras in einem realen Raum erfasst, werden die Nutzer jeweils an die gleiche Raumposition einer virtuellen 3-D-Szene versetzt. Der virtuelle Maschinenprototyp wird in dieselbe 3-D-Szene projiziert. Mithilfe der 3-D-Kameras ist ein volles Bewegungs-Tracking der Nutzer möglich. Die Anzahl der Kamerasysteme ermöglicht eine bedarfsgerechte Skalierung des Holodecks auf verschieden große Räume. Mithilfe sogenannter 3-D-Virtual-Reality-Brillen kann sich jeder Nutzer komplett kabellos frei im Raum bewegen und individuell beispielsweise die Produktionsszene oder die Maschine erkunden. Weiterhin sind die Brillen so angepasst, dass diese eindeutig einem Benutzer zugewiesen werden können. Datenhandschuhe ermöglichen eine individuelle Interaktion mit dem System. Der Datenhandschuh stellt nicht nur eine performante Lösung zur Nutzereingabe dar, sondern ermöglicht erstmals die für einen Menschen intuitivste Weise mit seiner Umwelt zu interagieren. So können beispielsweise Objekte mit der Hand gegriffen, im Raum verschoben und abgelegt werden. Systeminteraktionen können über unterschiedliche Gesten realisiert werden. Unterschiedliche Interaktionsszenarien werden hier iterativ erprobt und auf deren Nutzerakzeptanz geprüft. Durch den im Consumer-Bereich ausgelösten Technologietrend im Bereich Virtual Reality sind diese HardwareElemente prinzipiell für den professionellen Einsatz nutzbar und können auf dieser Basis optimiert werden.

Jedes lokale Holodeck wird über eine zentrale Holodeck-Workstation kontrolliert. Diese übernimmt die Synchronisierung der Bewegungsdaten aller lokalen Nutzer im Raum sowie die Bildsynchronisierung. Wesentliche Anforderung ist, die Latenz- und Reaktionszeit minimal zu halten. Ein charakteristisches Leistungsmerkmal ist dabei die sogenannte 


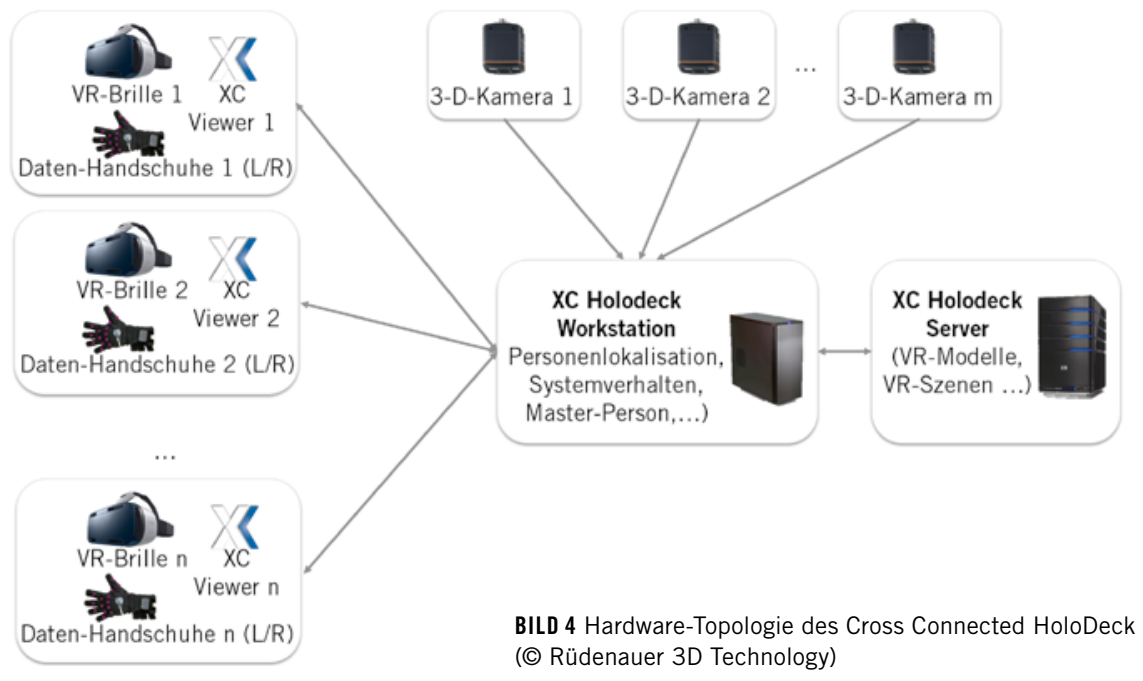

Motion-to-Photon-Latenz, welche die Zeitdauer vom Auslösen eines Bewegungssignals durch den Nutzer bis zur Darstellung dieser Bewegung in einer neu gerenderten 3-D-Szene beschreibt. Somit beschreibt die Motion-to-PhotonLatenz die Summe aller im VR-System auftretenden Latenzen. Eine hohe Latenz kann sich besonders negativ auf das Wohlbefinden des Nutzers auswirken und zu Schwindelgefühlen führen [8]. Die Grenze liegt hier bei etwa 15 ms [9].

Durch die Verbindung der Workstations über einen Holodeck-Server können mehrere Workstations - und somit Standorte synchronisiert werden. Dies ermöglicht es, mehrere Nutzer an unterschiedlichen realen Standorten gleichzeitig in denselben virtuellen Raum zu versetzen, mit der Möglichkeit, dass diese dort miteinander interagieren können.

\section{ANWENDUNGSSZENARIEN UND NUTZEN}

$\mathrm{Zu}$ einem Bruchteil der Kosten einer CAVE wird das Cross Connected HoloDeck für Anwendungen im Entwicklungs- und Schulungsbereich sowie für Ergonomieuntersuchungen optimiert. Maschinen können durch das Holodeck in übergreifenden Entwicklerteams virtuell im 3-D-Raum erlebt und Bauteile sowie Baugruppen auf Montierbarkeit an der virtuellen Montagelinie untersucht werden. Kostenintensive Iterationsschleifen von der Produktionslinie zurück über die Konstruktion können somit auf ein Minimum reduziert werden. Durch den Einsatz von Virtual-Reality-Technologien konnten in Studien bereits Zeiteinsparungen von bis zu 20 \% der Entwicklungszeit nachgewiesen werden [10]. Der virtuell aufgebaute Prototyp kann im Anschluss zur Schulung von Servicetechnikern und Maschinenbedienern eingesetzt werden. Neben schnelleren Instandsetzungszyklen führt das erhöhte Verständnis des Maschinenverhaltens zu einer Reduktion von ungeplanten Ausfällen aufgrund von Wartungsfehlern und Fehlbedienungen. Beim internationalen Einsatz der Maschinen spielt dies eine wesentliche Rolle. Sprachbarrieren und kulturelle Unterschiede stellen häufig eine große Hemmschwelle dar. Gleichzeitig führt das verbesserte Verständnis des Zusammenhangs zwischen Maschinenbedienung und dem, was in der Maschine passiert, zu einer effizienteren Führung der Maschine im Betrieb selbst.

Darüber hinaus liefern derartige virtuelle Schulungssituationen eine ideale Voraussetzung, um Ergonomieaspekte bei Bediener- und Servicetätigkeiten gemeinsam mit dem Endnutzer abzusprechen und das Feedback direkt über das virtuelle Modell in die Entwicklung zurück einfließen zu lassen. Bedienerschnittstellen einer Maschine können somit im virtuellen Raum erlebt, bewertet und iterativ optimiert werden, bevor ein Produkt überhaupt existiert.

\section{ZUSAMMENFASSUNG}

Das Cross Connected HoloDeck ist das erste kosteneffiziente Virtual-RealitySystem, das mehrere Nutzer standortübergreifend miteinander vernetzt. Im
Vergleich mit am Markt bestehenden Virtual-Reality-Lösungen kann zusätzlich das individuelle 3-D-Erlebnis jedes Nutzers deutlich gesteigert werden. Durch die Portabilität des Systems können global verteilte Stakeholder in kürzester Zeit an das virtuelle Produkt herangeführt und interaktiv in Entwicklungsprozesse eingebunden werden. Gerade diese Mobilität ermöglicht es Maschinenherstellern neue Geschäftsmodelle in der Entwicklung zu etablieren. Es bietet unter anderem die Möglichkeit, Produkttests in frühen Phasen zum Kunden zu verlagern. Zeitaufwendige Dienstreisen und langwierige Abstimmungsprozesse werden reduziert. Nicht zuletzt sind die Anschaffungskosten gegenüber heute bestehenden Systemen mit ähnlicher Funktionalität (zum Beispiel CAVE) erheblich reduziert. Das Cross Connected HoloDeck ebnet VirtualReality-Technologien den Weg in die Industrie, um über den gesamten Produktlebenszyklus Mehrwert zu schaffen.

\section{LITERATURHINWEISE}

[1] Christophers, M.: Entwicklung eines Vorgehensmodells für die unternehmensübergreifende, virtuelle Produktentwicklung. FAST, Karlsruher Institut für Technologie, Masterarbeit, Co-Betreuer: Rüdenauer, A.; Betreuer: Geimer, M., Karlsruhe, 2014 [2] Schäppi, B.: Handbuch Produktentwicklung. München, Wien: Carl Hanser, 2005

[3] Fraunhofer-Institut für Systemtechnik und Innovationsforschung (ISI): Untersuchung über die Zukunft der Produktion in Deutschland. Karlsruhe: Fraunhofer ISI, 2003

[4] Lu, S. C-Y.: A scientific foundation of collaborative engineering. CIRP Annals - Manufacturing Technology, Volume 49

[5] Verband der Automobilindustrie: Pressemitteilung zur Studie FAST 2025 - Massiver Wandel in der automobilen Wertschöpfungsstruktur. www.vda. de/de/meldungen/news/20121114-2.html., zugegriffen am 12.02.2013

[6] Rank, R.: Off-Highway hat andere Innovationszyklen. In: ATZoffhighway, Juli 2015, S. 20-25 [7] Knapp, O.: Design-to-Value: Increasing Product Profitability. Stuttgarter Gespräche 2011, Stuttgart, 2011

[8] Dörner, R.; et. Al.: Virtual und Augmented Reality (VR/AR): Grundlagen und Methoden der Virtuellen und Augmentierten Realität. Berlin/Heidelberg: Springer Vieweg, 2013

[9] Abrash M: Latency - the sine qua non of AR and VR. http://blogs.valvesoftware.com/abrash/latencythe-sine-qua-non-of-ar-and-vr/, 2012, zugegriffen am 17.12.2015

[10] Kells, D.; et al.: Virtually no limit to the exciting things VR can do... . In: raconteur.net - Sonderausgabe Raconteur, 17.12.2015: Virtual Reality, S. 6-7, zugegriffen am 17.12.2015 